\title{
Reportagem sôbre o D.A.S.P., realizada pelo Journal Français du Brésil (n. 160, 8eme année, Ier Féorier, 1959)
}

O Journal Français du Brésil, n. ${ }^{\circ} 160$, de fevereiro de 1959 , publicou, sob o titulo: Le Journal Français vous presente: M. Gerard Pic, professeur d'Administration Publique au D.A.S.P. uma reportagem, realizada por $M$. Jean Bazin, que temos o prazer de reproduzir para nossos leitores.

- Voyons, M. PIC qu'est-ce qui vous a le plus frappé depuis que vous êtes ici, il y a quelques mois, dans ce milieu de fonctionnaires qui composent le D.A.S.P.? («Departamento do Serviço Público»).

- C'est la gentillesse brésilienne, cher monsieur. Dès mon arrivée, je puis dire que mes nouveaux collègues, tous et toutes, se sont coupés en quatre pour me faciliter les choses.

- Par exemple?

- Oh! je vous donnerai un exemple qui n'a rien à voir avec le service. Je n'étais. pas ici depuis huit jours quand un fonctionnaire s'est offert à me faire visiter la ville, il m'a emmené successivement au Corcovado, au Pain de Sucre, au Jardin Botanique.

- Et vous avez ainsi découvert les merveilles touristiques de la «Cidade Maravilhosa»?

- Découvert? de visu, oui. Car avant d'embarquer, j'avais déjà lu dans plusieurs auteurs ayant passé à Rio les mêmes descriptions mirifiques qui m'avaient paru manquer de variété. Mais de visu, j'ai été vraiment ebahi.

- J'ai eu la même impression que vous quand j'ai débarqué à Rio, il y a 38 ans, en 1920.

- Savez-vous qu'après cette première excursion, je n'ai pas tardé à recevoir une nouvelle invitation, et pour ne pas déplaire à mon nouvel ami carioca, j'ai du feindre la plus grande suprise en remontant au Pain de Sucre, au Corcovado, mais à cette seconde fois, j'ai découvert la Vista Chinesa.

- Ca ne suffisait pas, il y avait encore d'autres attractions naturelles.

- Une troisième fois, ce fut un de mes élèves, du cours que je fais au D.A.S.P., qui s'offrit à être mon cicerone. Impossible de refuser. Bien m'en prit. Mon èlève avait une voiture. Après le Corcovado, il m'a emmené par une route de montagne à la Tijuca, et au retour, nous sommes 
redescendus dans la Zone Sud, par la Table de l'Empereur, d'où l'on a une vue vraiment resplendissante. De là, une nouvelle promenade au Jardin Botanique pour finir sur la plage.

— Et vous avez encore eu d'autres «guides»?

- Mais oui, en feignant ne pas connaitre, on finit par avoir tout vu. J'ai encore découvert au Jardin Zoologique, ces milliers d'oiseaux multicolores qui paraissent sortir d'un conte de fées. Maintenant je passe par une longue accalmie, il y cinq mois que je suis ici, et ma mission tire à sa fin. Mes collègues du D.A.S.P. estiment que je suis devenu un vrai carioca. C'est bien de l'honneur qu'ils me font.

Mon interlocuteur a été chargé de mission par le Quai d'Orsay en vertu des accords de Coopération Technique franco-brésilienne. M. de Fontenay, directeur de l'E.N.A. (Ecole Nationale d'Administration) avait été envoyé au Brésil en 1957 pour négocier ces accords d'engagement réciproque ayant pour objet des échanges de boursiers et une aide mutuelle à se prêter entre functionnaires et technicens des deux pays, spécialistes des PTT, ingénieurs des Ponts et Chaussées, des Eaux et Forêts des Mines, etc. Ces accords furent signés le 2 Septembre 1957.

M. Gerard PIC, arrivé en Aout dernier, en mission au près du D.A.S.P., est immédiatement entré en contact avec M. João Guilherme dE ArAgão, directeur du D.A.S.P. qui l'a chargé de professer un cours d'Administration Publique à l'«Escola do Serviço Público».

Le professeur Pic est un ancien élève de l'E.N.A., une école spéciale qui a profondément modifié la vie administrative en France. Entré à 23 ans, en 1949, sorti 3 ans après. De 1952 à 1958, resté à l'école même. En 1948, il avait reçu une affectation aux Affaires Indigènes du Maroc, et exerça aussi comme contrôleur civil adjoint à Meknès. Un renseignement en passant:

- Il y avait 70.000 fonctionnaires français au Maroc en 1956, me dit M. Pic. La plupart ont du être rappelés, il ne reste plus que des techniciens, ingénieurs des Ponts et Chaussées, agronomes, alors que nos fonctionnaires ont passé leurs fonctions aux Marocains. Le Maroc envoie des stagiaires à l'E.N.A., pour les former aux tâches administratives. C'est ainsi qu'un de mes élèves marocains à l'E.N.A., est devenu ministre plénipotentaire du gouvernement chérifien.

Après son stage au Maroc et ses études à l'E.N.A., M. Pic a été faire un stage dans l'industrie de la soierie à Lyon (1950). En 1951, il était au ministère des Finances à l'administration civile où il est resté de 1952 à 1958, tout en étant maitre de conférences a l'E.N.A.

Je sonde mon interlocuter sur l'Administration Française, il me répond, la main sur le coeur:

- Malgré une réputation injustifiée, l'Administration, chez nous, a ses défauts et ses qualités. Pendant les continuelles crises de cabinets de la IV République, on a toujours dit, dans la presse, pour rassurer l'opinion publique, que ces crises n'empêchaient pas l'Administration de continuer à gouverner le pays. C'etait un slogan erroné. En 1957 et 58, j'étais conseiller technique au cabinet du ministre des Finances, Pfimlin. En bien! 


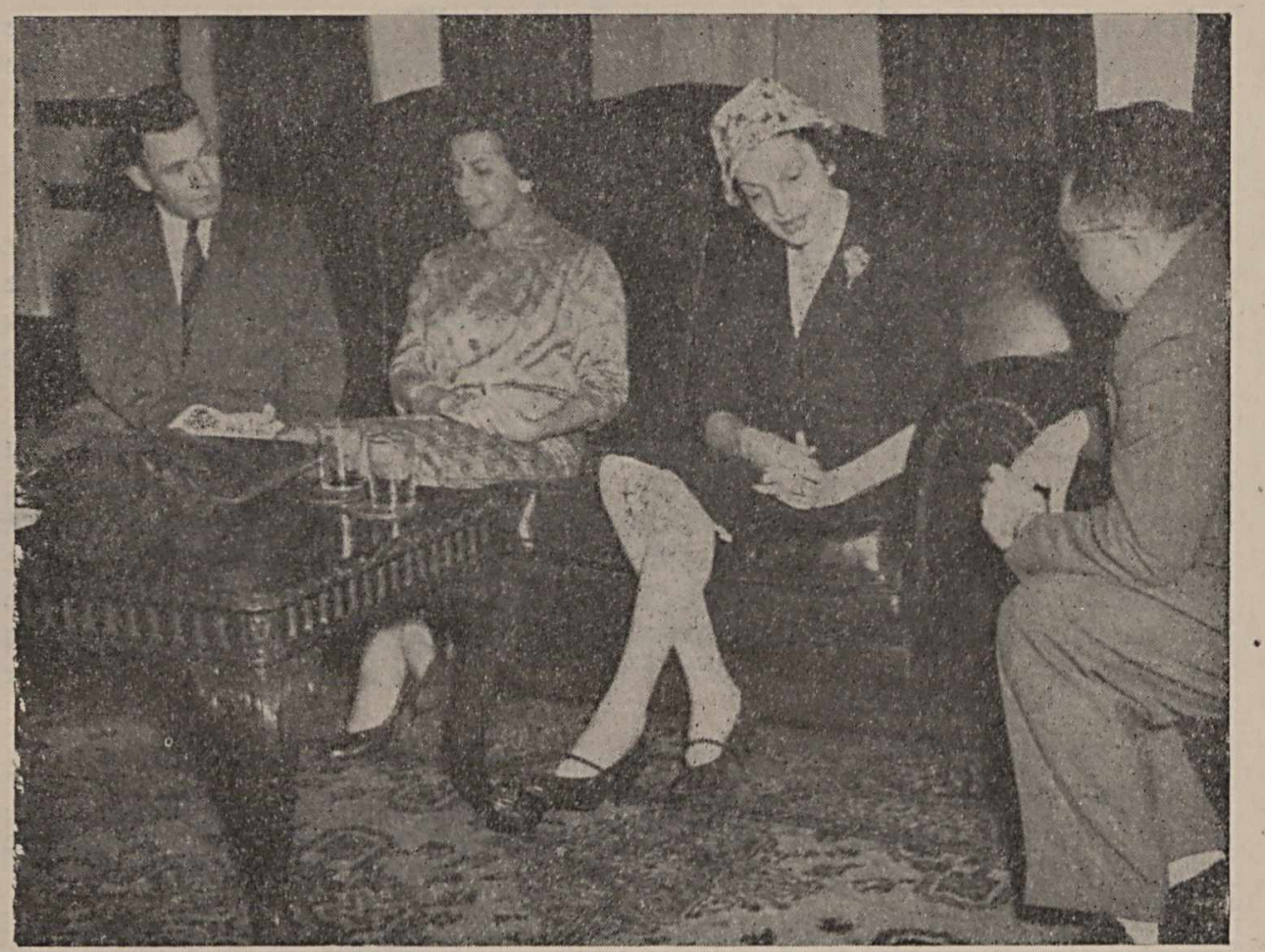

on se rendait compte que sans ministre, l'Administration ne pouvait fonctionner; car un ministère a plusieurs directions qui prennent des décisions collectives au conseil de cabinet du ministre, ce dernier agit en arbitre. En son abscence, les directions de chaque ministère sont sans arbitre et sans liaison, tout reste en suspens. Je vous citerai un exemple. La direction du Personnel devait 200 millions de crédits aux PTT. La direction du Budget ayant dit: non, la direction du Personnel rétorqua: alors, je vais avoir une grève. Il a fallu pour l'éviter prendre les 200 millions sur les disponibilités accessoires du budget, ce qui devait accroitre le déficit. Dans un tel cas, seul le ministre alors démmisionaire pouvait résoudre.

Ces crises ont révélé la nécessité d'une autorité permanente. La IV République avait de bons ministres, et a eu de bons Premiers, mais ils se voyaient paralysés par la faiblesse de leur majorité et les défections lors des votes de confiance, qui provoquaient les crises.

\section{QU'EST-CE QUE LE DASP ?}

J'interroge ensuite M. Pic sur ce «D.A.S.P.» que pour le grand public parait être une sorte de cénacle administratif hermétique seulement fréquenté par des initiés. On entend partout parler du D.A.S.P. et personne, parmi les simples citoyens, ne sait ce que c'est le D.A.S.P. alors qu'il s'agit d'un organisme de la plus haute importance ayant sous sa juridiction les centaines 
de milliers de fronctionnaires de tous les Etats et municipes du Brésil. C'est ainsi que j'ai eu l'honneur d'être présenté par M. Gérard Pic à João Guilherme de Aragão, directeur du D.A.S.P., qui s'exprime et écrit dans un français presque parfait et m'a accueilli avec cette «gentillesse brésilienne» qui aura si fort impressionné $M$. Pic à son arrivée, et qui pour nous, français devenus cariocas, fait partie de la «condition humaine» de ce grand pays.

M. Guilherme de Aragão est un jurisconsulte de Droit Administratif, il a publié plusieurs ouvrages très documentés et qui jettent une grande clarté sur un sujet aussi aride, pour ne citer que «La Juridiction Administrative au Brésil» et «Le D.A.S.P. et la fonction publique au Brésil» edités en français par «Imprensa Nacional». Sous la direction de M. Guilherme de Aragão, le président Juscelino Kubitschek a donné au D.A.S.P. une position de prestige qui a assuré sa consolidation définitive dans le cadre des institutions administratives du pays.

La Constitution de 1934 avait créé le Conseil Fédéral du Service Civil que celle de 1937 remplaça car le «Departamento do Serviço Público» (D.A.S.P.) qui commença à fonctionner en 1938 dans l'édifice du Ministère du Travail, tout en dépendant directement de la Présidence de la République, et finalement dans le building du Ministère des Finances depuis 1944. Il y a eu dans son organisation une double influence, tout d'abord nord-américaine et ensuite française.

Le D.A.S.P. a élaboré le Statut des Fonctionnaires avec leurs droits et leurs devoirs. Afin de protéger l'Etat contre les idéologies, la loi N. ${ }^{\circ} 1.802$ de 1953 a défini les crimes contre l'Etat et l'ordre public et social, prévoyant à son article 18 , des peines de 10 mois à 2 ans de prison aux fonctionnaires qui feraient cesser le fonctionnement du Service Public au moyen d'une action collective.

Le Statut, qui est plus tolérant qu'en France où toute activité professionnele privée et lucrative est interdite aux fonctionnaires, autorise l'activité privée des serviteurs publics en dehors du service sans que cela puisse porter atteinte a leurs obligations publiques et il ne faut pas mettre le service public au profit d'un intérêt personnel. D'autre part, le cumul de fonctions publiques est prohibé.

Tout fonctionnaire a le droit de demande ou de pétition, lui permettant de présenter un recours à son sujet ou sur des actes administratifs irréguliers, recours hiérarchique pouvant aller jusju'au Président de la République et qui doit comporter réponse dans un délai de 30 à 90 jours.

Le Statut définit également la-responsabilité des fonctionnaires qui peut être triple, tout d'abord disciplinaire et légal, d'accord avec les délits prévus par le Code Pénal brésilien: détournement de livres, documents et fonds; concussion, exaction, corruption, contrebande, prévarication, complicité criminelle, violence arbitraire, violation du secret professionnel, etc. Il peut y avoir deux instances, l'instance disciplinaire dont le jugement revient au D.A.S.P., et l'instance pénale dont la compétence revient à la justice fédérale. A ce sujet, la doctrine brésilienne «est d'accord avec la doctrine française, quand elle marque comme critérium de distinction entre la responsabilité disciplinaire et la responsabilité pénale, le fait qu'en droit pénal on 
applique le principe «nullum crimen nulla poena sine lege» ce qui n'arrive pas en droit disciplinaire».

La troisième responsabilité est civile, prévue intialement par la Constitution de 1891 et finalement par celle de 1946, responsabilité vis-à-vis de l'Adminis-' tration et des particuliers. Le procès administratif est le moyen adopté pour vérifier les fautes disciplinaires des fonctionnaires et leur appliquer les sanctions sont les chefs de service. Le Statut prévoit l'arrestation administative préventive et suspensive et la suspension préventive de 30 à 90 jours, et plus.

Le Statut du D.A.S.P. a fini par légaliser la situation des surnuméraires, en les titularisant dans certaines conditions et après un certain nombre d'années de service, et en les admettant ensuite au bénéfice de la retraite. Les sur numéraires ont les mêmes droits, de demande, procès administratif, recours, que les fonctionnaires. Mais le personnel des Travaux Publics ne jouit d'aucune garantie statutaire ou de droit public, n'ayant droit ju'au congé annueI et au repos hebdomadaire, d'accord avec la législation travailliste, et pouvant être dispensé suivant les nécessités du moment. Il en est de même pour le Personnel des Services et Charges diverses des tâcherons.

Dans la juridiction administrative fédérale, nul ne peut être nommé fonctionnaire s'il n'a subi avec succès un concours ouvert par le D.A.S.P. qui procède au classement des candidats et à leur approbation.

L'organe central compétent pour prendre toute dernière décision sur tous sujets administratifs d'orbite fédéral ets le D.A.S.P. et la plus haute autorité consultative est le «Consseiller général de la République» du Pouvoir Exécutif.

Enfin, le D.A.S.P. est un organe indépendant par rapport à tous les services administratifs. Il se maintient à l'écart des ministres en vertu de la nature de ses fonctions touchant à l'administration générale. Le D.A.S.P. a déjà maintes fois fait preuve de son indépendance, ce qui lui a attiré maintes critiques.

\section{L'ORGANISATION DU D.A.S.P.}

Le conseiller juridique du D.A.S.P. est M. Clenicio Duarte da Silva, adjoint a M. J. Guilherme de Aragão, directeur général. Le D.A.S.P. qui comprend les cinq division suivantes, ayant chacun leur directeur respectif:

I - Division du Personnel (Réglementation, Discipline, Traitements) : M. Waldyr dos Santos. II - Division du Budget (Evoluation des recettes et des dépenses, Service d'organisation) Dr. Barsante dos Santos. III - Division de Sélection (Organisation des concours. Fixation des programmes) Dr. Mario Lopes. IV - Division des Cours: $\mathrm{M}^{110}$ Estela de Souza Pessanha. $\mathrm{V}$ - Division des Edifices Publics (Construction des immeubles fédéraux Entretien) M. Luiz Phelippe de Barros. VI - Service d'Administration: M. José Medeiros. VII - Service de Documentation: M. Bandeira de Mello.

\section{LE COURS D'ADMINISTRATION PUBLIQUE}

Jesuis présenté à $\mathrm{M}^{11 \mathrm{e}}$ Estela de Souza Pessanha, chamante et si jeune qu'elle personnifie à la Direction de la Division des Cours que la valeur n'attend pas le nombre des années. C'est sous sa responsabilité que s'éla- 
borent les normes des dievrses formations professionnelles exigées par le Service Public et de plus, elle dirige les cours de «l'Ecole du Service Public» où notre compatriote, M. Gérard professe actuellement un cours d'Administration Publique.

A ce sujet, M. Pic me fait part d'une doléance de maintes fonctionnaires brélisiens, de la génération la plus âgée, qui apprirent parfaeitement le français et se plaignent n'avoir plus guère d'occasion de le parler cependant que les jeunes ne le parlent plus du tout, et cela bien, que le cours de M. Pic soit fréquenté par des jeunes qui malgré tout suivent la matière et montrent, par leurs réponses, qu'ilis ont compris.

Le Cours d'Administration Publique qui aura duré sept mois environ, (M. Pic rentre à Paris, fin Février) comporte quatre parties. Les problèmes humains, tout d'abord (entrée au Service Public, rémunération, développement des carrières, et la sélection pour l'avancement; obligations des fonctionnaires et sanctions; les relations humaines de l'Etat employeur avec ses serviteurs; les relations des fonctionnaires avec l'usager des services publics. La seconde partie est celle des moyens matériels: problèmes immobiliers, construction, financement, entretien; les services interministériels et autres; les problèmes mobiliers, concurrence des prix, acquisition, délais de livraison, association de l'Etat avec l'entreprise; les procédures d'administration. La troisième partie est celle des moyens financiers prévision des dépenses d'administration publique, application des dépenses, mécanisation, cout et rendement des services publics - La quatrième partie, celle des moyens d'organisation du pouvoir central, des services locaux, des problèmes de liaison; des problèmes de méthode de travail dans l'Administration publique, de leur rationalisation.

Entre autres, une impression de M. Pic au sujet d'une de ses élèves: «Une jeume femme assiste à mon cours avec sa fillete, de 5 à 7 ans. A la fin d'une séance, la petite s'endort et sa mère l'emporte dans ses bras en passant devant mon bureau. Taquin, je m'adresse à cette dame en lui faisant remarquer que le sommeil de sa fille ne flattait guère l'auteur du cours. Et la mère de me répondre, comme opur s'excuser: «Monsieur, elle est si petite».

M. Pic me dit encore:

_ On se fait à Paris une fausse idée de l'état d'espirit des Brésiliens. Ainsi, on n'avait dit que «le Brésilien n'aime pas que l'on considère son pays comme un pays tropical. Ne portez pas de vétements clairs et, sortout, ne vous plaignez jamais de la chaleur», m'avait-on recommandé. Or, dès notre première entrevue, le Directeur général du D.A.S.P. me demanda si je ne souffrais pas trop de la chaleur. Il faisait plus de $35 .^{\circ}$ Stoiquement, oubliant la sueur qui m'inondait, je lui reppondis:

- Mais, non, il ne fait pas chaud.

J'ai eu l'impression à ce moment que M. Guilherme de Aragão m'a pris pour un plaisantin. 